TITLE: Isentrope Energy, Hugoniot Temperature, and the Mie-Gruneisen Equation of State

AUTHOR: Charles A. Forest, DX-10, LANL

Los Alamos, NM 87545

SUBMTTED TO: 1995 APS Topical Conference on "Shock Compression of Condensed Matter" August 13-18, 1995 - Seattle, WA

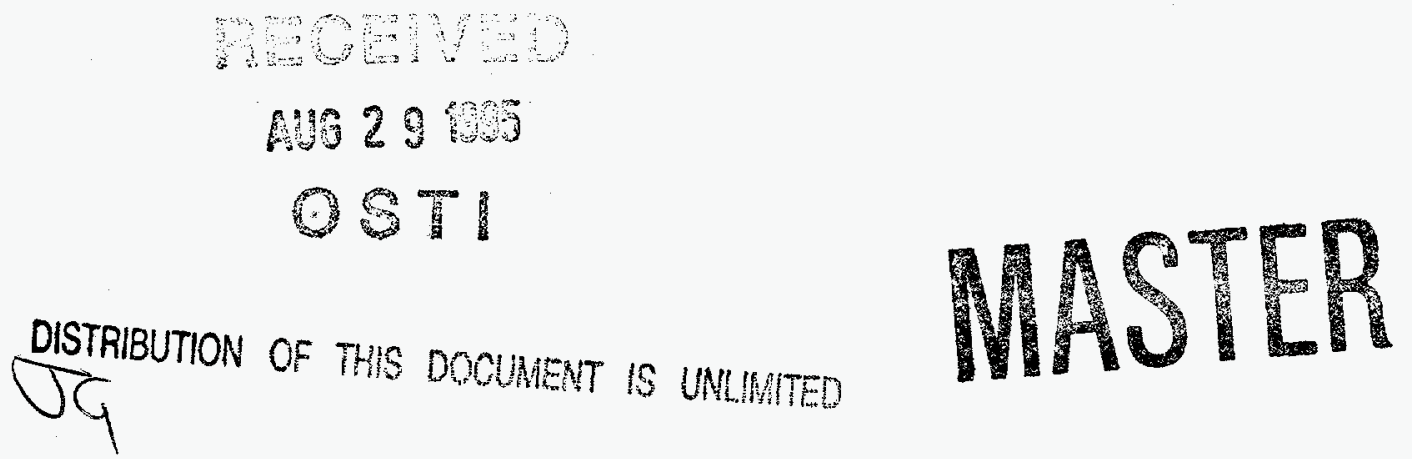

By acceptance of this article, the publisher recognizes that the U.S. Government retains a nonexclusive, royalty-free license to publish or reproduce the published form of this contribution, of to allow others to do so, for U.S. Government purposes.

The Los Alamos National Laboratory requests that the publisher identify this article as work performed under the auspices of the U.S. Department of Energy. 


\section{DISCLAIMER}

This report was prepared as an account of work sponsored by an agency of the United States Government. Neither the United States Government nor any agency thereof, nor any of their employees, makes any warranty, express or implied, or assumes any legal liability or responsibility for the accuracy, completeness, or usefulness of any information, apparatus, product, or process disclosed, or represents that its use would not infringe privately owned rights. Reference herein to any specific commercial product, process, or service by trade name, trademark, manufacturer, or otherwise does not necessarily constitute or imply its endorsement, recommendation, or favoring by the United States Government or any agency thereof. The views and opinions of authors expressed herein do not necessarily state or reflect those of the United States Government or any agency thereof. 


\section{DISCLAIMER}

Portions of this document may be illegible in electronic image products. Images are produced from the best available original document. 


\title{
ISENTROPE ENERGY, HUGONIOT TEMPERATURE, AND THE MIE-GRUNEISEN EQUATION OF STATE
}

\author{
Charles A. Forest \\ Group DX-I, MS P952, Los Alamos National Laboratory, Los Alamos, NM 87545
}

Analytic expressions for both the isentrope energy and temperature along the Hugoniot curve may be expressed in terms of a single integral function for a Mie-Gruneisen equation of state with constant heat capacity $c_{v}$.

\section{INTRODUCTION}

The Mie-Gruneisen equation of state with a Hugoniot reference line is commonly used in calculations. The temperature on the Hugoniot and isentrope curves are at times desired. Calculation of the temperature along the Hugoniot was given by Walsh and Christian (1), the expression for which contains an integral function. This integral, for constant heat capacity $c_{v}$, and general Gruneisen parameter $\Gamma(v)=v(\partial p / \partial e)$, will be shown to be that integral which is necessary for the calculation of isentropic energy.

The calculation of temperature or the calculation of an arbitrary isentrope is often useful in fluid dynamic modeling. These properties are used in mixture equations of state that assume pressure equilibrium along with temperature equilibrium or isentropic behavior following a first shock. There is a convenience in having the single integral function $I(u)$ that facilitates both purposes.

\section{BASIC FORMULATION}

The Mie-Gruneisen equation of state using a first-shock Hugoniot reference line is written

$$
p(v, e)=(\Gamma / v)\left(e-e_{h}(v)\right)+p_{h}(v)
$$

where $v=$ specific volume, $e=$ specific internal energy, $p_{h}(v)=$ Hugoniot pressure, $e_{h}(v)=$ $\frac{1}{2}\left(p_{h}(v)+p_{0}\right)\left(v_{0}-v\right)+e_{0}$, the Hugoniot relation.

The function $p_{h}(v)$ is calculated from two Rankine-Hugoniot relations (conservation of mass and momemtum) and the empirical $U_{s}(u)$ relation (the shock velocity into undisturbed material). These equations are, as functions of the shock particle velocity $u, v_{h}(u)=v_{0}\left(U_{s}(u)-u\right) / U_{s}(u)$, $p_{h}(u)=\rho_{0} u U_{s}(u)+p_{0}$, and $U_{s}(u)=c+s u+q u^{2}$.

Letting $u_{h}(v)$ be the inverse $v_{h}(u)$, then by composition $p_{h}(v)=p_{h}\left(u_{h}(v)\right)$.

The empirical $U_{s}(u)$ relation is represented piecewise by a linear or quadratic over $n$ segments, $u_{1}<u_{2}<\ldots<u_{n+l}$. A corresponding set of oppositely ordered volume segments are defined, $v_{1}>v_{2}>\ldots>v_{n+1}$ with $v_{j}=v_{h}\left(u_{j}\right)$. If $U_{s}(u)=$ $c+s u+q u^{2}$ on a segment, then $u_{h}(v)$ is obtained by solution of the quadratic equation

$$
\left(\frac{q u_{1}(v)}{c}\right) u^{2}-u+u_{1}(v)=0 \text { where }
$$




$$
u_{1}(v)=c\left(v_{0}-v\right) /\left(v_{0}-s\left(v_{0}-v\right)\right)
$$

If $q=0$, then $u_{h}(v)=u_{l}(v)$.

If $q \neq 0$, the two roots for $u_{h}(v)$ are

$$
\begin{aligned}
& u_{a}=2 u_{l}(v) /\left(1+\sqrt{1-4 q u_{l}(v)^{2} / c}\right) \text { and } \\
& u_{b}=c /\left(q u_{a}\right) .
\end{aligned}
$$

The root $u_{a}$ has the correct limit for $q=0$ and has good numerical precision; and for $q \neq 0$, the $u_{b}$ root has similar precision. Both are calculated using the fact that if the equation $a x^{2}+b x+c=0$ has roots $x_{1}$ and $x_{2}$, then $c=a x_{1} x_{2}$. The proper root must be selected to lie in the segment determined by $v$.

The derivatives of $p_{h}(v)=p_{h}\left(u_{h}(v)\right)$ with respect to specific volume $v$, or of any other function parametrically given as a function of $u$, requires the derivative $d u_{h} / d v$, which can be calculated implicitly from the mass equation

$$
U_{s}\left(u_{h}(v)\right) v=v_{0}\left[U_{s}\left(u_{h}(v)\right)-u_{h}(v)\right]
$$

Differentiation gives

$$
\frac{d u_{h}}{d v}=\frac{U_{s}\left(u_{h}(v)\right)}{\frac{d U_{s}}{d u}\left(v_{0}-v\right)-v_{0}}
$$

Using this result, with $u=u_{h}(v)$,

$$
\frac{d p_{h}}{d v}=\rho_{0}\left[U_{s}+\frac{d U_{s}}{d u} u\right] \frac{d u_{h}}{d v}
$$

An expression for the Gruneisen gamma $\Gamma$ in terms of $c_{v}, T, \beta$, and $c^{2}$ can be obtained from two thermodynamic equations related to $\Gamma$,

$$
c_{p}=c_{v}(1+\beta \Gamma T) \text { and } c_{p}=\beta c^{2} / \Gamma \text {, where }
$$

$$
c_{p}=(\partial e / \partial T)_{p}+p(\partial v / \partial T)_{p}, c_{v}=(\partial e / \partial T)_{v}
$$

$$
\beta=(\partial v / \partial T)_{p} / v, \text { and } c^{2}=(\partial p / \partial p)_{s}
$$

Equating the two $c_{p}$ expressions and solving the resulting quadratic equation for $\Gamma$ gives

$$
\Gamma=\frac{2\left(\beta c^{2} / c_{y}\right)}{\left.1+I 1+4\left(\beta c^{2} / c_{v}\right) \beta T\right]^{\frac{1}{2}}}
$$

\section{ISENTROPE ENERGY AND MIE- GRUNEISEN EOS}

From the thermodynamic equation, $T d s=d e+$ $p d v$ (with $d s \equiv 0$ ) and the equation of state,

$$
\frac{d e_{i}}{d v}=-p\left(v, e_{i}(v)\right)
$$

where $e_{i}(v)$ is the isentrope energy. The differential equation for $e_{i}$ is then

$$
\frac{d e_{i}}{d v}+(\Gamma / v) e_{i}=(\Gamma / v) e_{h}(v)-p_{h}(v)
$$

with initial value $e_{i}\left(v_{l}\right)=e_{1}$. Let now $g(v)=$ $(\Gamma / v)$, and let $G(v)=\exp \left\{\int g(v) d v\right\}$ be the integrating factor.

For two special cases of $(T / N)$, these functions are as follows:

1. If $\Gamma=\Gamma_{0}$, then $g(v)=(\Gamma / v)$ and $G(v)=v^{\Gamma}$.

2. If $\Gamma / v=\Gamma_{0} / v_{0}$, then $g(v)=\rho_{0} \Gamma_{0}$ and

$$
G(v)=\exp \left\{\rho_{0} \Gamma_{0} v\right\}
$$

Now multiply equation (2) by $G$ and integrate,

$$
\begin{gathered}
e_{i}(v) G(v)-e_{l} G\left(v_{l}\right)= \\
\int_{v_{l}}^{v} G(v)\left[g(v) e_{h}(v)-p_{h}(v)\right) d v
\end{gathered}
$$


Note that $\frac{d G}{d v}=G(v) g(v)$ and integrate by parts

$$
\int_{v_{l}}^{v} G g e_{h} d v=\left[G e_{h}\right]_{v_{l}}^{v}-\int_{v_{1}}^{v} G \frac{d e_{h}}{d v} d v .
$$

Also note

$$
\frac{d e_{h}}{d v}+p_{h}=\frac{1}{2} \frac{d p_{h}}{d v}\left(v_{0}-v\right)+\frac{1}{2}\left(p_{h}-p_{0}\right)
$$

Finally then

$$
\begin{gathered}
e_{i}(v) G(v)-e_{l} G\left(v_{l}\right)=G(v) e_{h}(v)-G\left(v_{l}\right) e_{h}\left(v_{l}\right) \\
-\int_{v_{l}}^{v} \frac{G(v)}{2}\left[\frac{d p_{h}}{d v}\left(v_{0}-v\right)+p_{h}-p_{0}\right] d v
\end{gathered}
$$

The remaining integral is not expressible in terms of elementary functions, and is not easily and accurately fit as a function of volume $v$ because $p_{h}(v)$ has a singular point at $v=v_{0}(s-1) / s$ when $U_{z}=c+s u$. Recall that the particle velocity has no such trouble and can be used for the integration change of variable. Thus let

$$
I(v)=\int_{v_{0}}^{v} \frac{G}{2}\left[\frac{d p_{h}}{d v}\left(v_{0}-v\right)+p_{h}-p_{0}\right] d v
$$

(note lower limit of integration). Changing integration variable, let

$$
v=v_{h}(u) \text { and } d v=\frac{d v_{h}}{d u} d u
$$

This particular choice of variable change is advantageous because $p_{h}(v)=p_{h}\left(u_{h}(v)\right)$ and

$$
\frac{d p_{h}}{d v}=\frac{d p_{h}}{d u} \frac{d u_{h}}{d v}=\rho_{o}\left(U_{s}+u \frac{d U_{s}}{d u}\right) / \frac{d v_{h}}{d u}
$$

Also note

$$
\left(v_{0}-v\right)=v_{0}^{u / U_{s}}, p_{h}-p_{0}=p_{0} u U_{s}, \text { and }
$$

$$
\frac{d v_{h}}{d u}=\frac{v_{o}\left[u \frac{d U_{s}}{d u}-U_{s}\right]}{U_{s}^{2}} .
$$

Then

$$
\begin{gathered}
I(u)=\int_{0}^{u} \frac{G\left(v_{h}(u)\right)}{2}\left[\frac{d p_{h}}{d v} \frac{d v_{h}}{d u}\left(v_{0}-v\right)+\right. \\
\left.\left(p_{h}-p_{0}\right) \frac{d v_{h}}{d u}\right] d u, \text { and finally, } \\
I(u)=\int_{0}^{u} \frac{G\left(v_{h}(u)\right) u^{2}}{U_{s}(u)} \frac{d U_{s}}{d u} d u
\end{gathered}
$$

and

$$
\begin{gathered}
e_{i}(v) G(v)=e_{1} G\left(v_{1}\right)+e_{h}(v) G(v)-e_{h}\left(v_{1}\right) G\left(v_{1}\right)- \\
{\left[I\left(u_{h}(v)\right)-I\left(u_{h}\left(v_{1}\right)\right)\right]}
\end{gathered}
$$

Remark: $e_{i}(v)$ is the energy of the isentrope through the point $\left(v_{1}, e_{1} f^{\text {which }}\right.$ may be any point in the domain of $p(v, e)$. A common usage is the case where $e_{1}=e_{h}\left(v_{1}\right)$, that is the isentrope for a material element that has experienced a first sbock.

The function $l(u)$ is represented on each $\left(u_{j}, u_{j+1}\right)$ interval by the form $I(u)=$ $a_{1}+u^{m}\left(a_{2}+a_{3} u+a_{4} u^{2}+a_{5} u^{3}+a_{6} u^{4}\right)$. In the neighborhood of $u=0$, we let $m=3$, and elsewhere let $m=1$. For calculation of the derivative of $I(u)$, the exact expression

$$
\frac{d l}{d u}=G\left(v_{h}(u)\right) u^{2} \frac{d U_{s}}{d u} / U_{s}(u) \text { is used. }
$$

The use of component equations of state in a equilibrium mixture equation of state requires not only an accurate function value but an accurate derivative so that the iterative equilibrium solution method will converge quickly. To that end, the function $I(u)$ is fitted by fitting the derivative of 
$I(u)$ and $I(u)$ simultaneously. One need not use polynomials, any suitable analytically integrable basis function set would do.

\section{TEMPERATURE AND HUGONIOT TEMPERATURE}

The temperature as a function of $(v, e)$ is

$$
T(v, e)=\frac{\left(e-e_{h}(v)\right)}{c_{v}}+T_{h}(v)
$$

where $e_{k}(v)=$ Hugoniot energy and $T_{k}(v)=$ Hugoniot temperature, and $c_{v}=$ constant heat capacity.

The temperature on the Hugoniot $T_{h}(v)$ is calculated via Walsh and Christian's method (1).

They write on page 1554 ,

$$
c_{v} \frac{d T_{h}}{d v}+\left(\frac{\partial p}{\partial T}\right)_{v} T_{h}=\frac{1}{2} \frac{d p_{h}}{d v}\left(v_{0}-v\right)+\frac{1}{2}\left(p_{h}-p_{0}\right) .
$$

Changing variable to $(v, e), p(v, T)=p(v, e(v, T))$ and thus

$$
\left(\frac{\partial p}{\partial T}\right)_{v}=\left(\frac{\partial p}{\partial e}\right)_{v}\left(\frac{\partial e}{\partial T}\right)_{v}=(\Gamma / v) c_{v} .
$$

The differential equation is then

$$
\frac{d T_{h}}{d v}+\frac{\Gamma}{v} T_{h}=\frac{l}{2 c_{v}}\left\{\frac{d p_{h}}{d v}\left(v_{0}-v\right)+p_{h}-p_{0}\right\}
$$

with initial value $T_{h}\left(v_{0}\right)=T_{0}$. Using the same integrating factor and notation as in the integration of the isentrope energy equation,

$$
\begin{gathered}
T_{h}(v) G(v)-T_{0} G\left(v_{0}\right)= \\
\int_{v_{0}}^{v} \frac{G}{2 c_{v}}\left\{\frac{d p_{h}}{d V}\left(v_{0}-v\right)+p_{h}-p_{0}\right\} d v .
\end{gathered}
$$

For $c_{v}=$ constant, the integral is just $\left.l(v)\right) c_{y}$, and thus

$$
T_{h}(v) G(v)=T_{0} G\left(v_{0}\right)+\frac{1}{c_{v}} I\left(u_{h}(v)\right) .
$$

\section{ACKNOWLEDGEMENTS}

I especially want to thank Genevieve Vigil and Larry Hill for their help in preparing this manuscript.

\section{REFERENCES}

1. J. M. Walsh and R. H. Christian, Equation of State of metals from Shock Wave Measurements, The Physical Review, 97, No. 6, 1544-1556, March 15. 1955. 